\title{
Graphene Quantum Dots prepared by Electron Beam Irradiation for Safe Fluorescence Imaging of Tumor
}

\author{
Honghong Cao ${ }^{1,2,3,4}$, Wei Qi ${ }^{5}$, Xudong Gao ${ }^{6}$, Qiang $\mathrm{Wu}^{1}{ }^{凶}$, Longlong Tian ${ }^{2,3}{ }^{凶}$, Wangsuo $\mathrm{Wu}^{2,3}$ \\ 1. Lanzhou University Second Hospital, Lanzhou University, Lanzhou, 730000, China. \\ 2. Institute of National Nuclear Industry, Lanzhou University, Lanzhou, 730000, China. \\ 3. Frontiers Science Center for Rare Isotopes, Lanzhou University, Lanzhou, 730000, China. \\ 4. Lanzhou Resources \& Environment Voc-Tech University, Lanzhou, 730000, China. \\ 5. Hubei Key Laboratory of Bioinorganic Chemistry \& Materia Medica, School of Chemistry and Chemical Engineering, Huazhong University of Science and \\ Technology, Wuhan, 430000, China. \\ 6. CAS Key Laboratory of Chemistry of Northwestern Plant Resources, Institute of Chemical Physics, Chinese Academy of Sciences (CAS), Lanzhou, 730000, \\ China. \\ $\triangle$ Corresponding authors: E-mail: lzu2h_wq@126.com (Q. Wu), tianll12@lzu.edu.cn (L.Tian).
}

(C) The author(s). This is an open access article distributed under the terms of the Creative Commons Attribution License (https://creativecommons.org/licenses/by/4.0/). See http://ivyspring.com/terms for full terms and conditions.

Received: 2021.09.16; Accepted: 2021.10.16; Published: 2022.01.01

\begin{abstract}
Graphene quantum dots (GQD) have attracted much attention due to their unique properties in biomedical application, such as biosensing, imaging, and drug delivering. However, scale preparing red luminescing GQD is still challenging now. Herein, with the help of electron beam irradiation, a simple, rapid, and efficient up-to-down strategy was developed to synthesize GQD with size of $2.75 \mathrm{~nm}$ emitting $610 \mathrm{~nm}$ luminescence. GQD were further functionalized with polyethylene glycol (PEG) and exhibited good solubility and biocompatibility. The potential in vivo toxicity of PEGylated GQD could completely be eliminated by the clinic cholesterol-lowering drug simvastatin. PEGylated GQD could selectively accumulate in tumor after intravenous injection as a security, reliable and sensitive tumor fluorescence imaging agent. Therefore, this work presented a new method preparing red luminescing GQD for biomedical application.
\end{abstract}

Key words: Graphene quantum dots; Red luminescence; Electron beam irradiation; Tumor imaging

\section{Introduction}

Cancer is a major disease seriously threatening human life and health [1-3]. Early diagnosis is an effective method to reduce cancer mortality, improve the survival rate, and prolong life of patient $[3,4]$. Compared with the traditional diagnosis methods (such as dripping-blood chip detection [5], genetic testing [6], biopsy [7], computed tomography [8], B-ultrasound [9], magnetic resonance imaging [10]), fluorescence imaging is a low-cost, high-precision and non-invasive diagnostic technology [11]. Quantum dots as a kind of quasi-zero-dimensional nanomaterials break through the limitation of dimensionality, and showing high yield of fluorescence quantum, stable optical properties, and resistance to photobleaching [12]. Graphene quantum dots (GQD), as an emerging fluorescence nanomaterial, possess many excellent characteristics such as strong luminous intensity and stability, chemical redox inertness and easy chemical modification [13-17], showing great potential of applications in bioimaging, biosensor and drug deliverer $[18,19]$.

The existing preparation methods of GQD mainly focuses on the hydrothermal cutting method, oxidative cutting carbon fiber method, and electrochemical peeling method [20-22]. The hydrothermal cutting method is similar to the oxidative cutting carbon fiber method, and the method is a classic method for preparing GQD, but the method is relatively cumbersome, and a variety of strong acids are introduced [23]. The electrochemical stripping method requires a long time for pre-processing graphite, slow process of post-processing products, and low synthesis yield 
[24]. Therefore, it is still a great challenge for researchers to develop a simple, rapid, scale, and efficient preparation method of GQD.

Radiation synthesis technologies have attracted great attention due to its advantages, such as rapid, efficient and clean industrial potential $[25,26]$. The electron itself is a very strong reducing agent, so the extra chemical reductant does not need to be added during the synthesis processes [27]. Moreover, the morphology and size could be adjusted by controlling the radiation dose, which provides potential method for the preparing small-size GQD [28].

Therefore, in this study, radiation synthesis technology was successfully used to prepare red luminescence GQD with size of $2.75 \mathrm{~nm}$. GQD were further functionalized with polyethylene glycol (PEG) and exhibited good solubility and biocompatibility [29]. The potential in vivo toxicity of PEGylated GQD (PGQD) could completely be eliminated after injection of clinic cholesterol-lowering drug simvastatin (Supplementary Information Figure S1) $[15,16,29]$. PEGylated GQD were found to selectively accumulate in tumor after intravenous injection and could act as a sensitive tumor fluorescence imaging agent (Figure 1). Therefore, this work presented a new method preparing red luminescing GQD for biomedical application.

\section{Results and Discussion}

\section{Synthesis and characterizations of PGQD}

In this work, GQD were synthesized by cutting electron beams-irradiated graphite (Figure 2 and Figure S2) [30]. GQD were modified with PEG for improving its water solubility and biocompatibility [31], so the color of GQD and PEGylated GQD (PGQD) were black and yellowish brown, as shown in Figure 3a. Transmission electron microscope (TEM) images of PGQD shows a narrow size distribution between 1 and $5 \mathrm{~nm}$ with average size diameter of $\approx 2.75 \mathrm{~nm}$. The high-resolution TEM (HR-TEM) image (Figure 3d) indicates high crystallinity of the PGQD, with a lattice parameter of $0.24 \mathrm{~nm}$, which was the (002) lattice fringes of graphene [32-34]. The UV-vis and fluorescence spectra of the PGQD were shown in Figure 4a. An obvious absorption peak could be

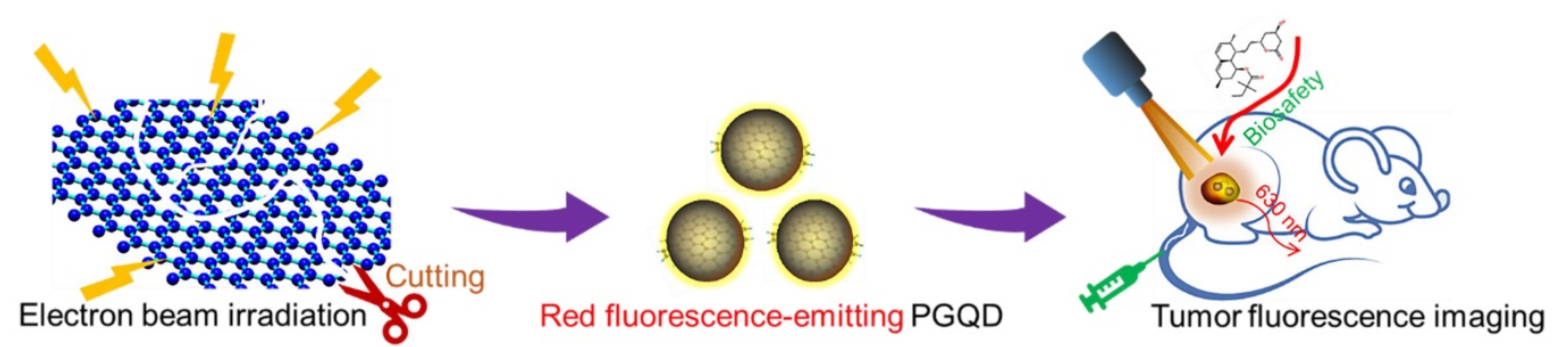

Figure 1. Schematic diagram of irradiation synthesis of graphene quantum dots for tumor fluorescence imaging.

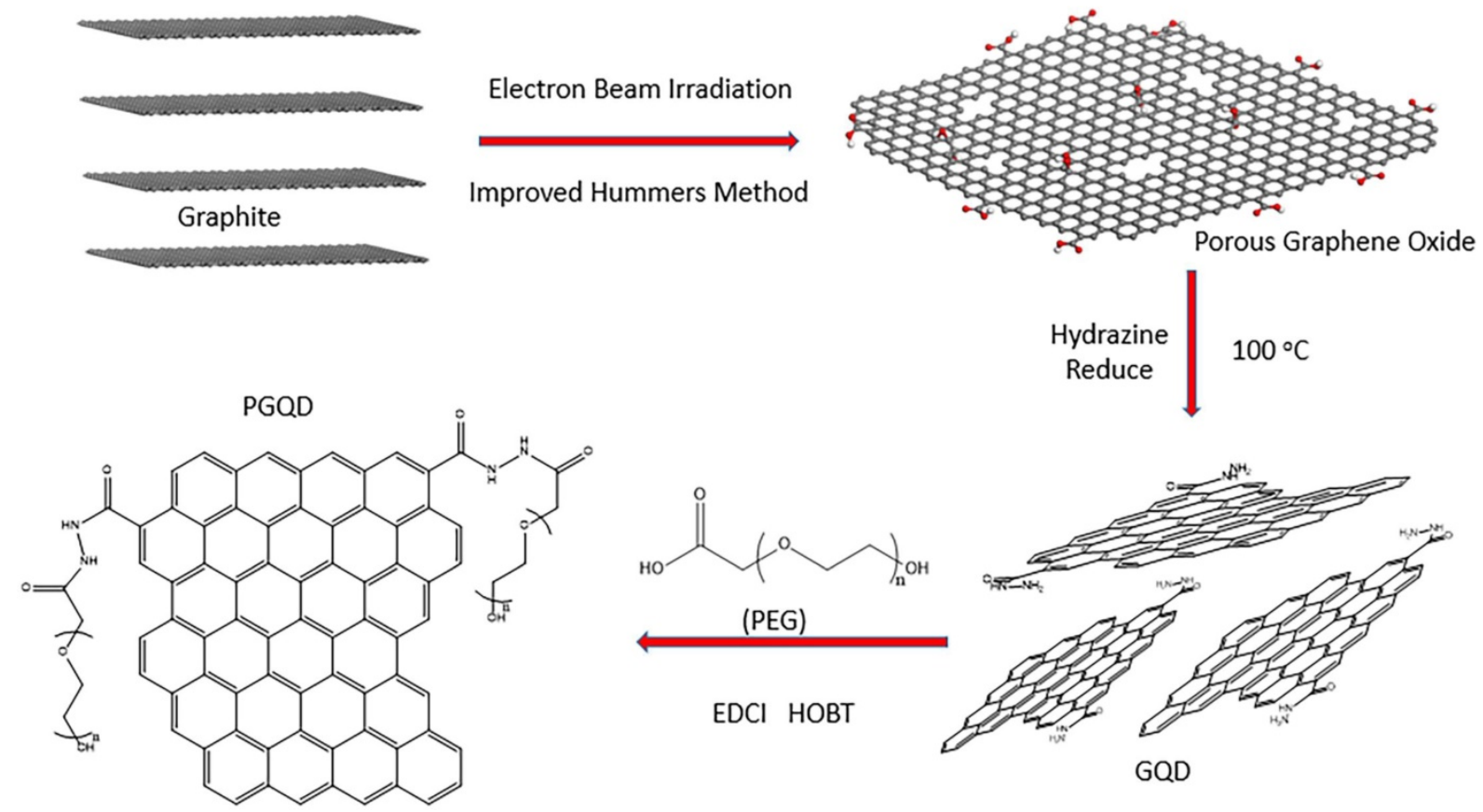

Figure 2. Schematic illustrating the fabrication process of GQD and PGQD. 
observed at $\approx 200 \mathrm{~nm}$, which could be attributed to $\Pi-\Pi^{*}$ transition from benzene structures, and the weak peak at $\approx 280 \mathrm{~nm}$ was from chemically reduced graphene $[35,36]$. When the excitation wavelength was changed from 320 to $420 \mathrm{~nm}$, fluorescenceemission spectra of PGQD shows the excitationdependent of photoluminiscence (PL) peak. With the increase of excitation wavelength, the intensity of PL peak increases rapidly, and the peak slowly shifted to long wavelengths, $\approx 6 \%$ quantum yield of the GQD could be obtained, suggesting that the yield was relatively high [37]. X-ray diffraction (XRD) of PGQD didn't show the typical broad (002) peak (Figure 4b), which could be attributed to the large amount of PEG coating on the surface of GQD. However, the strong D peak $\left(1386 \mathrm{~cm}^{-1}\right)$ and $\mathrm{G}$ peak $\left(1599 \mathrm{~cm}^{-1}\right)$ could be found in Raman spectra (Figure S3) of PGQD, which confirmed the feature structure [38-40]. As compared with the Fourier transform infrared (FTIR) spectrum of $\mathrm{GO}$, the strong and broad absorption peak at $\approx 3400$ $\mathrm{cm}^{-1}$ from the $\mathrm{O}-\mathrm{H}$ stretching vibration could be observed in PGQD and PEG [41]. There was a sharp adsorption at $2863 \mathrm{~cm}^{-1}$ in PGQD and PEG attributing to $\mathrm{C}-\mathrm{H}$; a peak of $\mathrm{C}=\mathrm{O}$ stretching vibration at $\approx 1719$ $\mathrm{cm}^{-1}$ could be seen clearly for GO and PGQD samples; a weak adsorption at $\approx 1385 \mathrm{~cm}^{-1}$ in PGQD indicates the presence of the $\mathrm{C}-\mathrm{N}$ bonds. Thermogravimetric analysis (TGA) from Figure 4d shows that PEG began to pyrolyze at $\approx 200^{\circ} \mathrm{C}$, but weight loss of PGQD starts at $\approx 300^{\circ} \mathrm{C}$. A sharp decalescence peaks could be seen

a

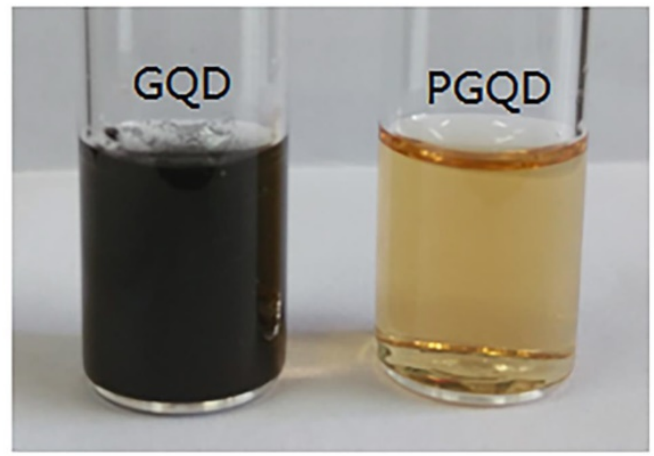

C

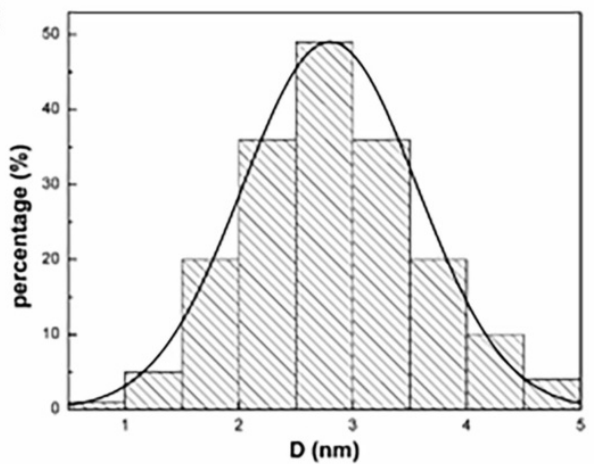

in Differential Scanning Calorimetry (DSC) of PGQD at $\approx 400{ }^{\circ} \mathrm{C}$, which could be attributed to the thermal decomposition of rest GQD. TGA and DSC results suggests that PEG has been functionalized covalently on the surface of GQD, and every PGQD average linked 20 PEG chains (Figure 4d). The characterization results showed that PGQD exhibits uniform size and excellent fluorescence properties.

\section{In vivo biodistribution of GQD and PGQD}

The labelling method was used to quantitatively study the biodistribution of GQD and PGQD in mice [42]. GQD and PGQD were labeled with 131I by chloramine $\mathrm{T}$ oxidation method. The radiolabeling yields and stability of GQD were determined by paper chromatography, with saline and acetone as mobile phase (Figure 4e). The radiolabeling yields were over $60 \%$. The distributions (Figure 5a-b) showed that GQD with low solubility were quickly captured by pulmonary capillary bed and mainly accumulated in lung. However, PGQD were found to accumulated in reticuloendothelial system and kidney, suggesting that PGQD with small size could also be excreted through the kidney. The distribution of GQD and PGQD were independent with simvastatin injection (Figure $5 c-d$ ), but the clearance rates of GQD and PGQD were obviously improved after simvastatin injection, especially in lung and spleen, which might reduce the in vivo toxicity of GQD and PGQD.

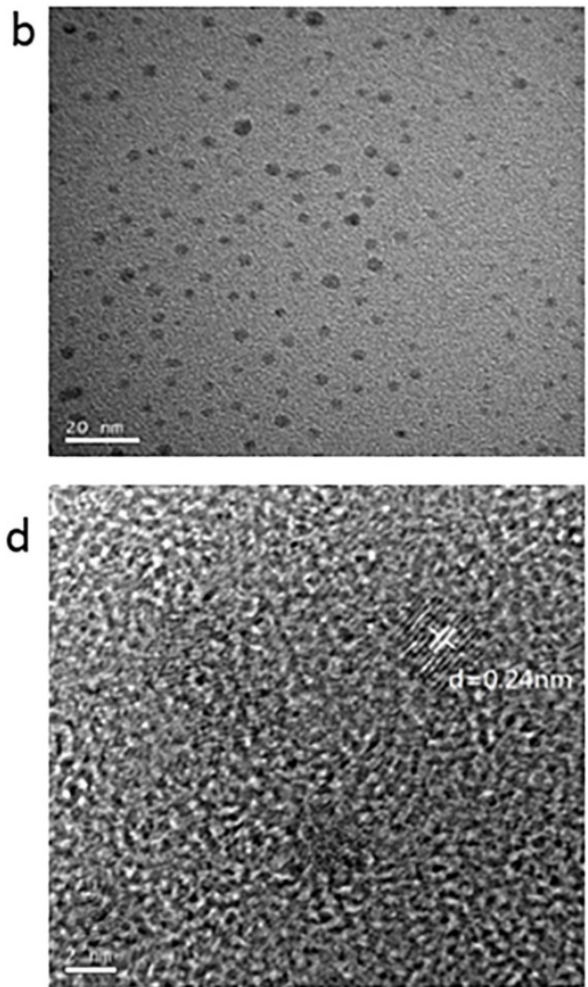

Figure 3. (a) $2 \mathrm{~g} / \mathrm{L}$ GQD and PGQD solution. (b) TEM image. (c) Size distribution. (d) HR-TEM image of PGQD. 

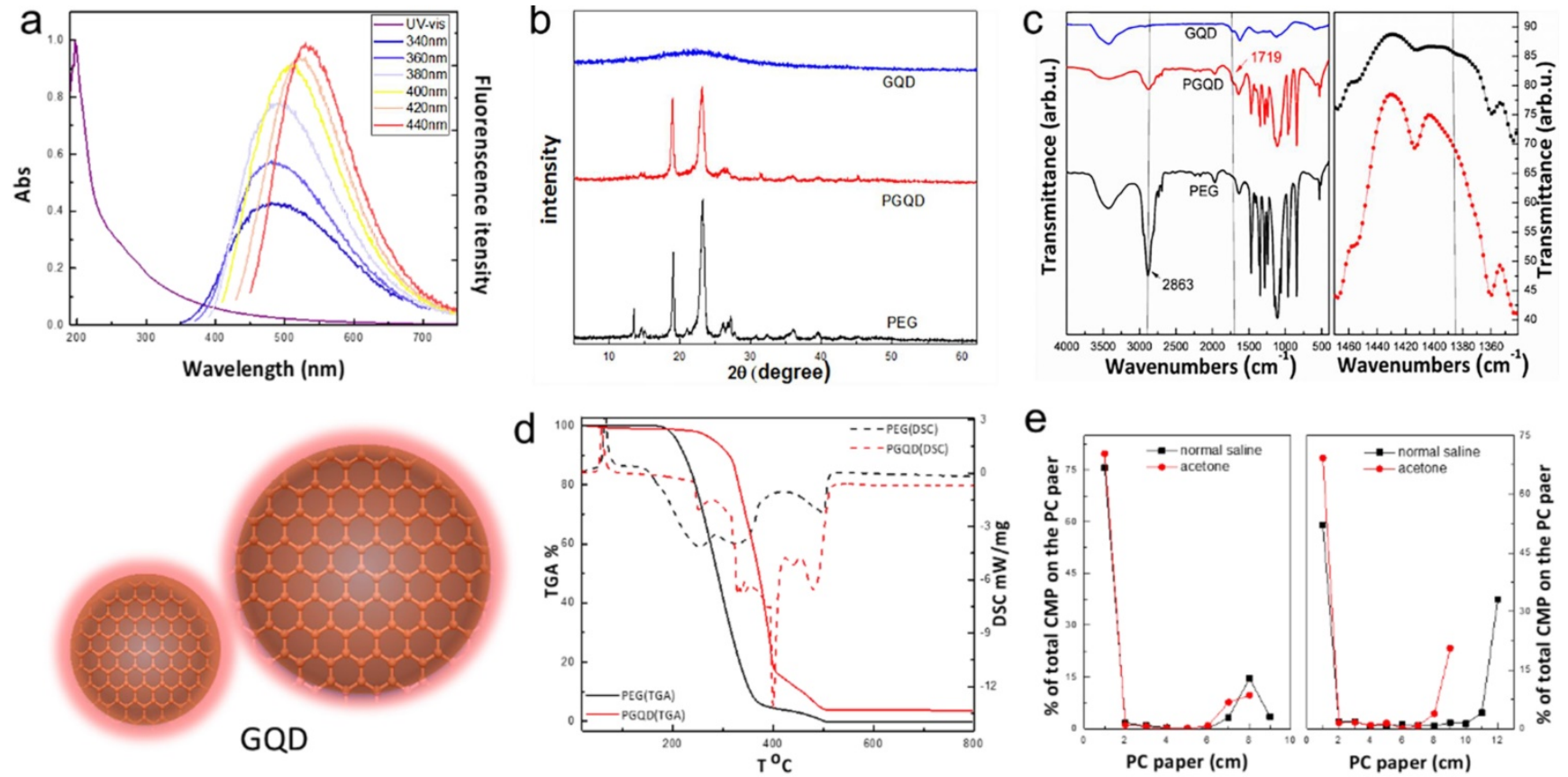

Figure 4. (a) UV-vis and PL spectra of PGQD. (b) XRD. (c) FT-IR of GQD, PGQD and PEG.(d) TGA and DSC of PGQD and PEG. (e) The determination radiolabling yields of GQD (left) and PGQD (right) by paper chromatography in normal saline and acetone.

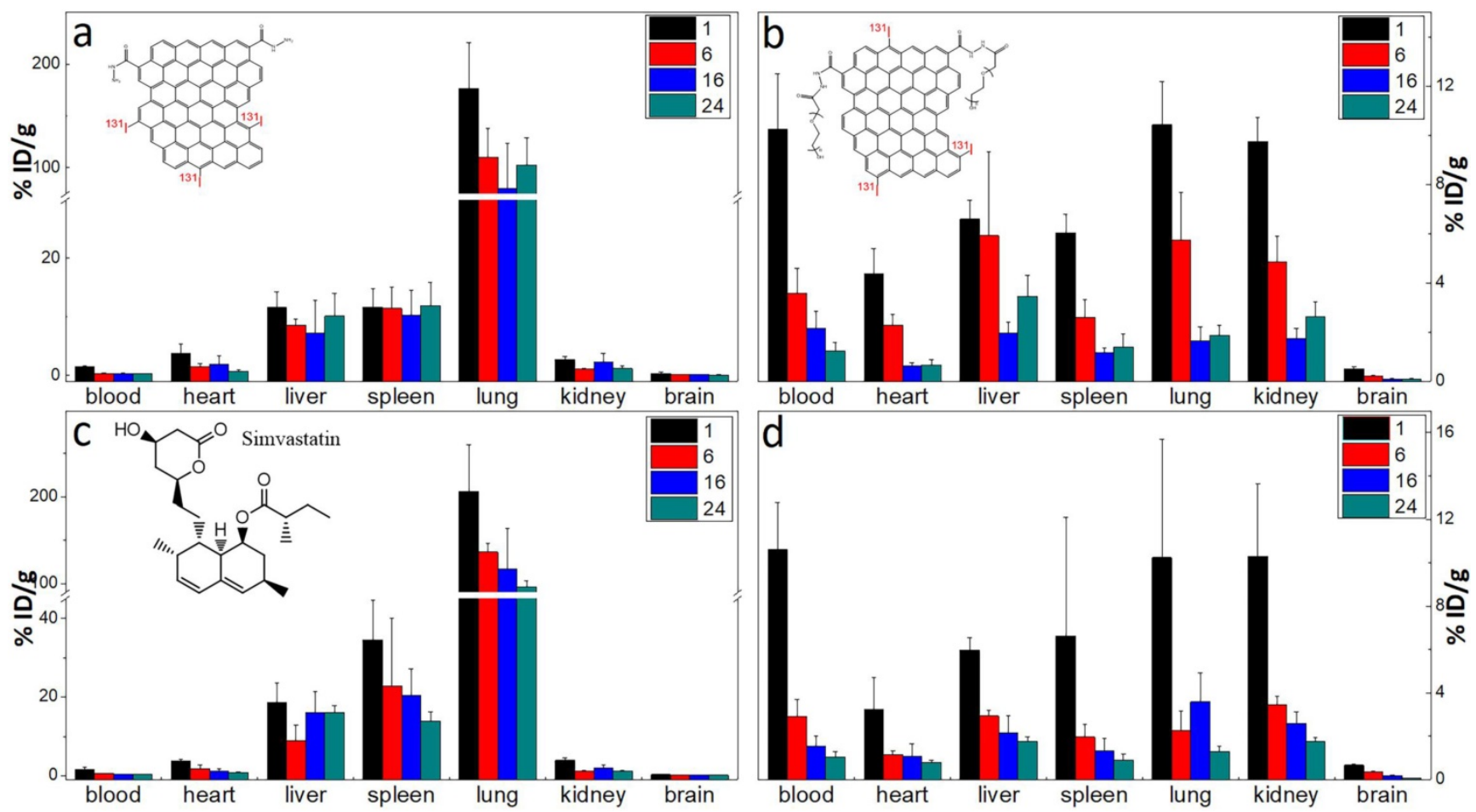

Figure 5. The biodistribution of GQD (a) and PGQD (b) the effect of simvastatins on the biodistribution of GQD (c) and PGQD (d) in mice at $1,6,16$ and $24 \mathrm{~h}$ post injection of ${ }^{|3|} \mid$-labeled materials.

\section{Serum biochemistry}

Serum biochemistry test (Figure 6a) was conducted to study the potential toxicity of GQD and PGQD in mice in vivo. As a sensitive indicator of kidney functions, the Cystatin C (Cys-C) in the blood increased. Some liver function markers were also tested, such as alanine aminotransferase (ALT), aspartate aminotransferase (AST) and total bilirubin (TBIL). The increase of AST and TBIL indicated hepatic toxicity after GQD and PGQD injection. Therefore, statins were used to eliminate the accumulation and toxicity of GQD and PGQD [41], so the injection sequence of simvastatin and nanoparticles was first investigated (Figure 6b) [43]. Simvastatin $(150 \mu \mathrm{g}, 10 \mathrm{mg} / \mathrm{kg})$ was injected at $5 \mathrm{~h}$ 
before, together with, and $5 \mathrm{~h}$ after the injection of GQDP/GQD $(400 \mu \mathrm{g}, 25 \mathrm{mg} / \mathrm{kg})$, then mice were sacrificed at $24 \mathrm{~h}$ for collecting blood (5 mice per group). Cys-C, AST and TBIL in the blood of mice treated by simvastatin and GQD together decreases significantly, indicating that simvastatin could improve the function of kidney and liver, and Cys-C, ALT, AST and C-reactive protein (CRP) in the blood of PGQD group mice decreases significantly after treatment of simvastatin. The results indicate that combined injection of simvastatin and GQD/PGQD could significantly alleviate the toxicity of GQD/PGQD in vivo.

The effect of simvastatin dose on toxicity in vivo was also investigated as shown in Figure 6c. Blood urea nitrogen (BUN), serum creatinine (CREA), ALT and AST of GQD increases unexpectedly by injection of simvastatin with $200 \mu \mathrm{g}$ dose, which may be attributed to the drug toxicity of simvastatin. However, importantly, almost all biochemistry parameters have reached normal level for mice combined injected by PGQD with simvastatin, indicating that the toxicity of PGQD could be reduced efficiently by low dose of simvastatin. In general, 50 or $150 \mu \mathrm{g}$ simvastatin could reduce the toxicity of GQD/PGQD in vivo. Moreover, simvastatin, lovastatin, atorvastatin, fluvastatin were chosen to study the effect of statins types on the toxicity of GQD/PGQD in vivo (Figure 6d). Mice were injected by GQD/PGQD mixed with 50 or $150 \mu \mathrm{g}$ statins, then sacrificed at $24 \mathrm{~h}$ for collecting blood. Results show that all four statins all could be used to reduce the toxicity of GQD/PGQD in vivo.
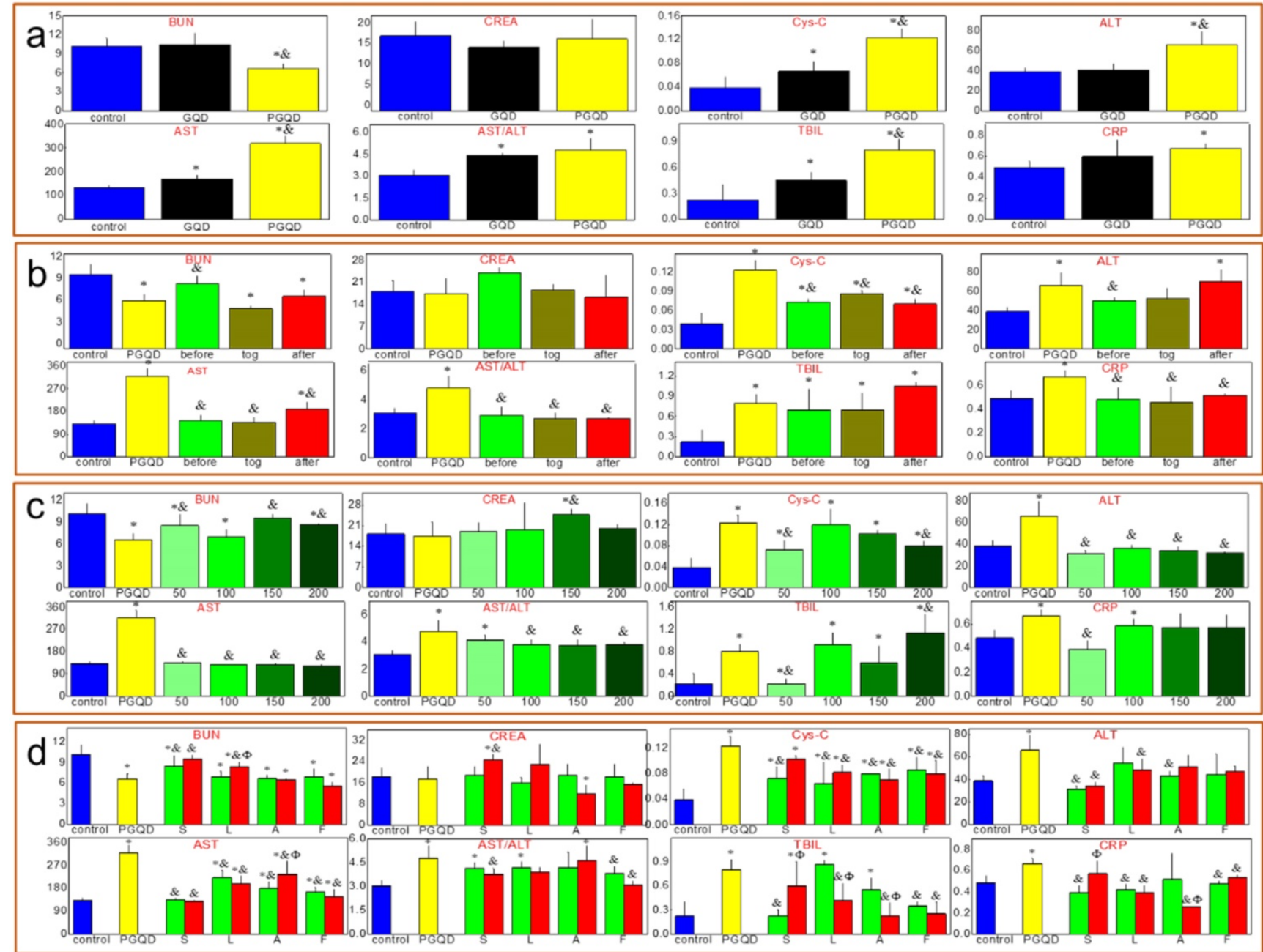

Figure 6. (a) Serum biochemistry test of mice injected normal saline (control group), GQD or PGQD. * $p<0.05$ compared with the control groups. \& $p<0.05$ compared with GQD group. $n=5-6$. (b) The effect of injection schedule of simvastatin and PGQD on toxicity. ${ }^{*}<<0.05$ compared with the control groups. \& $p<0.05$ compared with PGQD group. $n=5-6$. (c) Effect of dose of simvastatin on the toxicity of PGQD. ${ }^{*} p<0.05$ compared with the control groups. \& $<0.05$ compared with PGQD group. $n=5-6$. (d) Effect of four statins (dose of 50 and $150 \mu \mathrm{g}$ ) on the toxicity of PGQD. ${ }^{*}<<0.05$ compared with the control groups. \& $p<0.05$ compared with GQD group. ${ }^{\Phi} p<0.05$ compared the dose of $50 \mu g$ statins groups. $n=5-6$. 


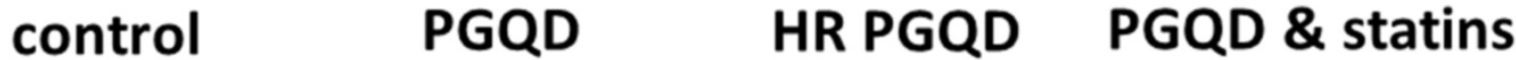

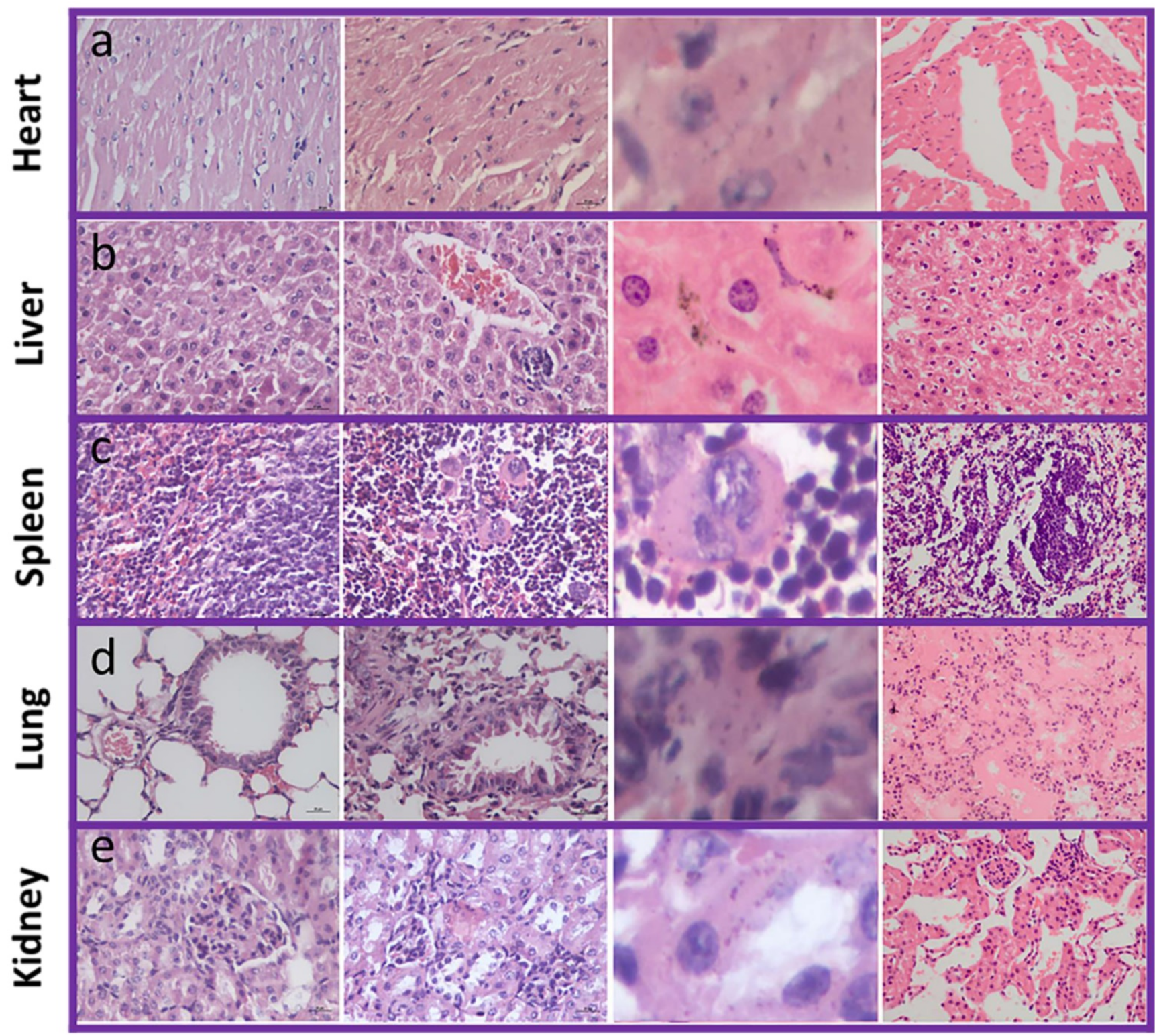

Figure 7. Photographs and high-resolution (HR) photographs of H\&E stained tissue slices (heart, liver, spleen, lung and kidney) of mice injected with of PGQD or PGQD mixed with simvastatin.

\section{Histology Examination}

To further confirm effect of statins on the toxicity of GQD/PGQD in vivo, the mice tissues were collected at $24 \mathrm{~h}$ after injecting with normal saline, GQD, PGQD, GQD with simvastatin, and PGQD with simvastatin for make slices of Haematoxylin and Eosin (H\&E) staining (Figure 7). Large numbers of nanoparticles were observed in the heart, liver, spleen, lung, and kidney tissues of GQD and PGQD group. After simvastatin injection, nanoparticles were eliminated from heart, liver and kidney and nanoparticles-induced damages could be repaired. Meanwhile, nanoparticles were observed much higher in lung than other tissues, which efficiently reduced after co-administration of simvastatin. Histology examination indicates that the nanoparticles accumulated in tissues could be efficiently cleared and tissue damage could be repaired after statins injection.

\section{Red blood cell (RBC) test}

The effect of nanoparticles on RBC has been investigated (Figure S4-5). Optical microscope and SEM images show that the morphology of RBCs remain unchanged after incubation with $100 \mu \mathrm{g} / \mathrm{mL}$ of GQD/PGQD for $4 \mathrm{~h}$ as compared with the control group, which indicates that toxicity of GQD and PGQD to RBCs was not significant. 

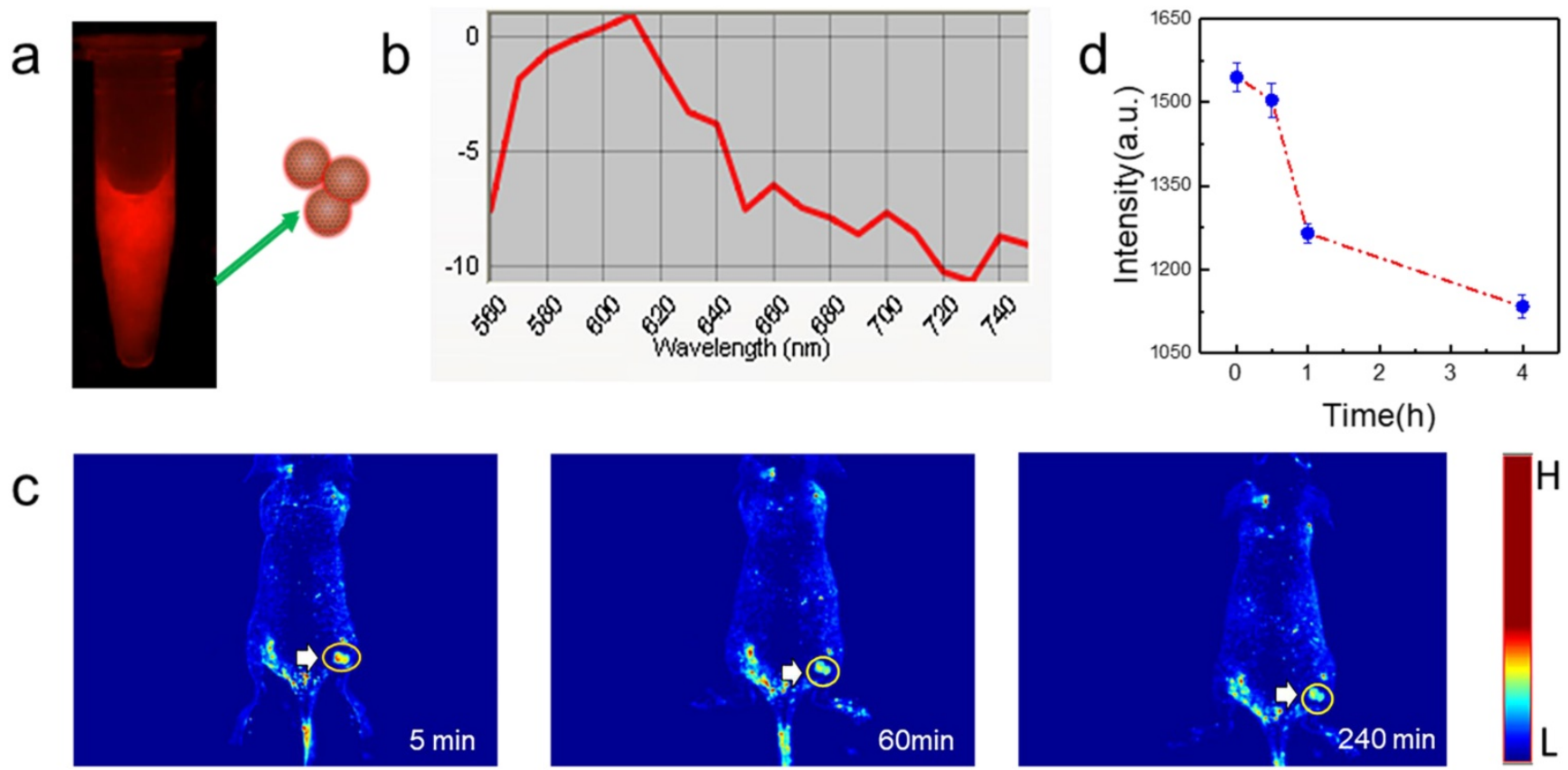

Figure 8. In vivo tumor fluorescence imaging with PGQD. (a) Fluorescence images of PGQD. (b) Fluorescence emission spectra of PGQD. (c) Mice bearing 4T1 tumors were intravenously injected with PGQD suspension solution and imaged with a small animal fluorescence imaging system at different time points (5 min, $1 \mathrm{~h}$ and $4 \mathrm{~h}$ ). (d) Fluorescence signals intensity in tumor.

\section{Fluorescence imaging of tumor}

PGQD could emit strong red fluorescence at about $610 \mathrm{~nm}$ (Figure 8a-b). Mice bearing 4T1 tumors were intravenously injected with PGQD suspension solution and imaged with a small animal fluorescence imaging system. After 5 minutes post injection, strong red fluorescence signals were observed in tumor, indicating that PGQD would selectively and rapidly accumulate in the tumor area (Figure 8c). The fluorescence signals in tumor (Figure 8d) were found to decrease with time, indicating that small size PGQD could be gradually eliminated. Those results proved that PGQD prepared by electron beam irradiation could was a good tumor-targeting imaging agent.

\section{Conclusion}

Graphene quantum dots were successfully prepared with electron beam irradiated graphite. The obtained GQD exhibited uniform size and excellent fluorescent property. GQD were further functionalized with polyethylene glycol (PEG) and exhibited good solubility and biocompatibility. The potential in vivo toxicity of PEGylated GQD (PGQD) could be completely eliminated after injection of clinic cholesterol-lowering drug simvastatin. PEGylated GQD were found to selectively accumulate in tumor after intravenous injection and could act as a sensitive tumor fluorescence imaging agent. This work presented a new method preparing red luminescing GQD for biomedical application.

\section{Materials and methods}

\section{Synthesis and characterization of GQD and PGQD}

In our previous work [44], heavily oxidized graphene oxide (HGO) with lots defects was prepared by the improved Hummers method with electron beam irradiated graphite. A $0.4 \mathrm{MeV}$ linear electron accelerator (USA, WASIK, $\mathrm{P}=16 \mathrm{KW}$ ) was used here. Before the GQDs preparation, the graphite flake (about $5 \mathrm{~g}$ ) was packaged by a $10 \times 10 \mathrm{~cm}$ PE bag and irradiated at 5 MGy with a dose rate of $8 \mathrm{kGy}$. GQD was obtained through directly reducing $\mathrm{HGO}$ by hydrazine at $100{ }^{\circ} \mathrm{C}$ for $24 \mathrm{~h} .200 \mathrm{mg}$ of carboxylated PEG (3350 Da) was activated with $20 \mathrm{mg}$ of 1-Ethyl3-(3-dimethyllaminopropyl) carbodiie hydrochlide (EDCI) in $50 \mathrm{mM}$ phosphate buffer ( $\mathrm{pH}$ 5.5). After 15 min, $20 \mathrm{mg}$ of GQD was added into the solution and further stirred for $2 \mathrm{~h}$ for complete reaction. At last, the solution was concentrated and rinsed with water through spin-dialysis (MWCD=8000-14000 Da) to obtain PGQD [45].

The prepared GQD and PGQD was collected and characterized by UV-Vis spectra, Fluorescence spectra, Transmission electron microscopy (TEM), X-ray Diffraction (XRD), Raman spectroscopy, Fourier transform infrared (FTIR) spectroscopy. UV-Vis spectra were collected with a PerkinElmer Lambda 35 spectrophotometer. Fluorescence emission spectra were recorded with a FLSP 920 fluorescence spectrometer (Edinburgh Instruments Ltd.). TEM was 
examined using a Tecnai-G2-F30Field Emission Transmission Electron Microscope (FEI Corporation). XRD was scanned from $5^{\circ}$ to $60^{\circ}$ on powder X-ray diffractometer (Panalytical). Raman spectra from 500 to $4000 \mathrm{~cm}^{-1}$ were collected on an in Via-Reflex Raman scope using a $632.8 \mathrm{~nm}$ He-Ne laser (Renishaw). FTIR spectra were recorded from 400 to $4000 \mathrm{~cm}^{-1}$ on a NEXUS 670 5-DX 170SX spectrometer (Nicolet Instrument Corporation).

\section{1| Labeling of GQD and PGQD}

The GQD (2 mg/mL) was labeled by ${ }^{131}$ I using a standard chloramine $\mathrm{T}$ oxidation method $[42,46,47]$. A mixture of $5 \mathrm{~mL}$ of GQD $(2 \mathrm{mg} / \mathrm{mL}), 1 \mathrm{mCi} \mathrm{Na}{ }^{131} \mathrm{I}$ and $100 \mu \mathrm{L} 4 \mathrm{mg} / \mathrm{mL}$ chloramine-T (Sigma-Aldrich) was reacted in a $\mathrm{pH} 7.5$ phosphate buffer $(0.02 \mathrm{M})$ for $30 \mathrm{~min}$ at room temperature. Excess ${ }^{131} \mathrm{I}$ was completely removed by centrifugation with water for $4 \sim 6$ times until no detachable gamma activity in the filtrate solution. The PGQD was labeled by the same method, while the excess ${ }^{131}$ I was completely removed with ultrafiltration centrifuge tube $(\mathrm{MWCO}=3 \mathrm{kDa})$ for $24 \mathrm{~h}$. Radiolabeling stability was tested with paper chromatography (saline and acetone as mobile phase).

\section{Biodistribution of 131 |-Labeled GQD and PGQD and the influence of simvastatin}

Kunming mice initially weighing $15 \mathrm{~g}$ to $18 \mathrm{~g}$ were provided by the Laboratory Centre for Medical Science, Lanzhou University, Gansu, China. All animals were housed in individual cages in a temperature-controlled $\left(21{ }^{\circ} \mathrm{C}\right.$ to $\left.22{ }^{\circ} \mathrm{C}\right)$ and light-controlled (turned on from 08:00 $\mathrm{h}$ to 20:00 h) environment and were fed food and tap water ad libitum. All animal protocols were in accordance with the European Communities Council Directive of November 24, 1986 (86/609/EEC) and approved by Institutional Animal Care and Use Committees of Gansu Province Medical Animal Center and Lanzhou University Animal Committees Guideline (China). The body weights of the mice were determined before they were injected intravenously (i.v.) with 20 $\mathrm{mg} / \mathrm{kg}$.bw of ${ }^{131} \mathrm{I}$-Labeled GQD or PGQD solution (injection volume of $0.2 \mathrm{ml}$, GQD and PGQD groups were diluted from $2 \mathrm{mg} / \mathrm{mL}$ to $0.8 \mathrm{mg} / \mathrm{mL}$ by saline, while the simvastatin groups were diluted from 2 $\mathrm{mg} / \mathrm{mL}$ to $0.8 \mathrm{mg} / \mathrm{mL}$ by $0.5 \mathrm{mg} / \mathrm{mL}$ simvastatin). Mice were sacrificed at 1, 6, 16 and $24 \mathrm{~h}$ after injection. Tissues from the heart, lungs, liver, spleen, kidney and brain were immediately dissected. Each tissue or organ was wrapped in foil, weighed and counted. Data were corrected for physical decay of radioactivity. The distribution in the tissues was presented in percent injected dose per gram of wet tissue (\% ID/g), which could be calculated by the percent injected dose (tissue activity/total activity dose) per gram of the wet tissue [48]. Statistics were based on standard deviations of $4 \sim 6$ mice per group.

\section{Blood biochemistry analysis and Histology Examinations}

Healthy female Kunming mice were injected with $500 \mathrm{uL}$ of $0.8 \mathrm{mg} / \mathrm{mL}$ GQD or PGQD (a dose of $20 \mathrm{mg} / \mathrm{kg}$ ) and statins was injected under different doses or sequence (before, together or after injecting GQD or PGQD). Mice were sacrificed at $24 \mathrm{~h}$ after injection. Other five healthy Kunming mice were used as the untreated control and sacrificed at $24 \mathrm{~h}$. An approximate $0.5 \mathrm{~mL}$ portion of blood from each mouse was collected for a blood biochemistry analysis before the mouse was euthanatized. The serum biochemistry data was measured in the Lanzhou University Second Hospital. Major organs (including liver, kidneys, spleen, heart and lung) from those mice were harvested and fixed in formalin for hematoxylin and eosin (H\&E) staining. Statistics were based on standard deviations of 5 mice per group.

\section{RBC test}

Fresh blood was obtained from Kunming mice, anticoagulated with heparin and centrifuged at 4000 $\mathrm{rpm}$ for $5 \mathrm{~min}$. The plasma was removed. The separated erythrocytes were washed three times by centrifugation ( $4000 \mathrm{rpm}, 5 \mathrm{~min}$ ) in 10 volumes of PBS. The supernatant and buffy coat of white cells were carefully removed with each washing. Washed erythrocytes were finally re-suspended with the same buffer and stored at $4{ }^{\circ} \mathrm{C}$ and used within $6 \mathrm{~h}$. The erythrocyte suspension was incubated with 100 $\mu \mathrm{g} / \mathrm{mL}$ of GQD, PGQD. At $4 \mathrm{~h}, 100 \mu \mathrm{L}$ of these mixtures were dropped onto a glass slide and observed by optical microscopy and confocal laser scanning microscope. The incubated suspensions were fixed with a glutaraldehyde solution (3\%), followed by centrifugation (4000 rpm, $5 \mathrm{~min}$ ), and then washed with gradient ethanol. Afterwards, the samples were vacuum-dried for SEM characterization [49].

\section{Fluorescence Imaging of Tumor}

Female nude mice were purchased from Nanjing Peng Sheng Biological Technology Co. Ltd. and used under protocols approved by Lanzhou University Laboratory Animal Center. $1 \times 10^{6} 4 \mathrm{~T} 1$ cells were suspended in $50 \mu \mathrm{L}$ PBS, and then injected subcutaneously into the back of each mouse. When the tumor volume reached about $100 \mathrm{~mm}^{3}$, the mouse was intravenously injected with PGQD and imaged with Maestro ${ }^{\mathrm{TM}}$ In-Vivo Imaging System at different time points post injection. 


\section{Statistical Analysis}

The experimental data is sorted by excel and reported data as mean values \pm SD of multiple determinations. All statistical analysis were carried out using SPSS software. Through the KplmogorovSmirnov Npar test and the homogeneity of variance test, the statistical significance in the difference was evaluated by analysis of variance (ANOVA) with the significance level of 0.05 or Kruskal-Wails-test method.

\section{Supplementary Material}

Supplementary figures.

http://www.ntno.org/v06p0205s1.pdf

\section{Acknowledgements}

This work was supported by the National Natural Science Foundation of China (No. 21974146 and 32101149), and the China Postdoctoral Science Foundation (no. 2020M671143). The authors thank the Opening fund of Hubei Key Laboratory of Bioinorganic Chemistry \& Materia Medica (No. BCMM202005).

\section{Author Contributions}

H.C. and L.T. conceived the project. H.C., Q.W., and L.T. performed the experiments and characterizations. H.C. and L.T. co-wrote the manuscript. All authors discussed the results and commented on the manuscript.

\section{Competing Interests}

The authors have declared that no competing interest exists.

\section{References}

1. Ancey PB, Contat C, Meylan E. Glucose transporters in cancer - from tumor cells to the tumor microenvironment. FEBS J. 2018; 285: 2926-43.

2. Bray F, Ferlay J, Soerjomataram I, Siegel RL, Torre LA, Jemal A. Global cancer statistics 2018: GLOBOCAN estimates of incidence and mortality worldwide for 36 cancers in 185 countries. CA Cancer J Clin. 2018; 68: 394-424.

3. Miller KD, Nogueira L, Mariotto AB, Rowland JH, Yabroff KR, Alfano CM, et al. Cancer treatment and survivorship statistics, 2019. CA Cancer J Clin. 2019; 69: 363-85.

4. McCormack V, Aggarwal A. Early cancer diagnosis: reaching targets across whole populations amidst setbacks. Br J Cancer. 2021; 124: 1181-2.

5. Caputo D, Papi M, Coppola R, Palchetti S, Digiacomo L, Caracciolo G, et al. A protein corona-enabled blood test for early cancer detection. Nanoscale. 2017; 9. 349-54.

6. Offit K, Tkachuk KA, Stadler ZK, Walsh MF, Diaz-Zabala H, Levin JD, et al. Cascading After Peridiagnostic Cancer Genetic Testing: An Alternative to Population-Based Screening. J Clin Oncol. 2020; 38: 1398-408.

7. Pinzani P, D'Argenio V, Del Re M, Pellegrini C, Cucchiara F, Salvianti F, et al. Updates on liquid biopsy: current trends and future perspectives for clinical application in solid tumors. Clin Chem Lab Med. 2021; 59: 1181-200.

8. Hu X, Sun J, Li F, Li R, Wu J, He J, et al. Renal-Clearable Hollow Bismuth Subcarbonate Nanotubes for Tumor Targeted Computed Tomography Imaging and Chemoradiotherapy. Nano letters. 2018; 18: 1196-204.

9. Farhadi A, Ho GH, Sawyer DP, Bourdeau RW, Shapiro MGJS. Ultrasound imaging of gene expression in mammalian cells. Science. 2019; 365: 1469-75.

10. Aghighi M, Theruvath AJ, Pareek A, Pisani LL, Alford R, Muehe AM, et al. Magnetic Resonance Imaging of Tumor-Associated Macrophages: Clinical Translation. Clin Cancer Res. 2018; 24: 4110-8.
11. Campbell E, Hasan MT, Gonzalez Rodriguez R, Akkaraju GR, Naumov AV. Doped Graphene Quantum Dots for Intracellular Multicolor Imaging and Cancer Detection. ACS Biomater Sci Eng. 2019; 5: 4671-82.

12. Chinnathambi S, Shirahata N. Recent advances on fluorescent biomarkers of near-infrared quantum dots for in vitro and in vivo imaging. Sci Technol Adv Mater. 2019; 20: 337-55.

13. Liu J, Zhang P, Yang X, Wang K, Guo Q, Huang J, et al. Aptamer-mediated indirect quantum dot labeling and fluorescent imaging of target proteins in living cells. Nanotechnology. 2014; 25: 505502.

14. Röding M, Bradley SJ, Nydén M, Nann T. Fluorescence Lifetime Analysis of Graphene Quantum Dots. J Phys Chem C. 2014; 118: 30282-90.

15. Kumar YR, Deshmukh K, Sadasivuni KK, Pasha SKK. Graphene quantum dot based materials for sensing, bio-imaging and energy storage applications: a review. RSC Adv. 2020; 10: 23861-98.

16. Singh H, Sreedharan S, Tiwari K, Green NH, Smythe C, Pramanik SK, et al. Two photon excitable graphene quantum dots for structured illumination microscopy and imaging applications: lysosome specificity and tissue-dependent imaging. Chem Commun. 2019; 55: 521-4.

17. Wang Z, Chen D, Gu B, Gao B, Liu Z, Yang Y, et al. Yellow emissive nitrogen-doped graphene quantum dots as a label-free fluorescent probe for $\mathrm{Fe} 3+$ sensing and bioimaging. Diam Relat Mat. 2020; 104: 107749-

18. Sun L, Xiong Z, Shen F, Wang Z, Liu Z. Biological membrane derived nanomedicines for cancer therapy. Sci China Chem. 2021; 64: 719-33.

19. Gu T, Chen T, Cheng L, Li X, Han G, Liu Z. Mesoporous silica decorated with platinum nanoparticles for drug delivery and synergistic electrodynamic-chemotherapy. Nano Res. 2020; 13: 2209-15.

20. Huang H, Yang S, Li Q, Yang Y, Wang G, You X, et al. Electrochemical Cutting in Weak Aqueous Electrolytes: The Strategy for Efficient and Controllable Preparation of Graphene Quantum Dots. Langmuir. 2018; 34: 250-8.

21. Ahirwar S, Mallick S, Bahadur D. Electrochemical Method To Prepare Graphene Quantum Dots and Graphene Oxide Quantum Dots. ACS Omega. 2017; 2: 8343-53

22. Zhao Y, Wu X, Sun S, Ma L, Zhang L, Lin H. A facile and high-efficient approach to yellow emissive graphene quantum dots from graphene oxide. Carbon. 2017; 124: 342-7.

23. Ma M, Hu X, Zhang C, Deng C, Wang X. The optimum parameters to synthesize bright and stable graphene quantum dots by hydrothermal method. J Mater Sci: Mater Electron. 2017; 28: 6493-7.

24. Marin S, Merkoi AJN. Direct electrochemical stripping detection of cystic-fibrosis-related DNA linked through cadmium sulfide quantum dots. Nanotechnology. 2009; 20: 055101.

25. Freitas de Freitas L, Varca GHC, Dos Santos Batista JG, Benévolo Lugão A. An Overview of the Synthesis of Gold Nanoparticles Using Radiation Technologies. Nanomaterials (Basel). 2018; 8: 939.

26. Fani M, Maecke HR, Okarvi SM. Radiolabeled peptides: valuable tools for the detection and treatment of cancer. Theranostics. 2012; 2: 481-501.

27. Yang Z, Zhu Y, Dong Z, Li W, Yang N, Wang X, et al. Tumor-killing nanoreactors fueled by tumor debris can enhance radiofrequency ablation therapy and boost antitumor immune responses. Nat Commun. 2021; 12: 4299.

28. Čubová K, Čuba V. Synthesis of inorganic nanoparticles by ionizing radiation - a review. Radiat Phys Chem. 2020; 169.

29. Columbus S, Painuly D, Nair RP, Krishnan VK. Role of PEGylated CdSe-ZnS quantum dots on structural and functional properties of electrospun polycaprolactone scaffolds for blood vessel tissue engineering. Eur Polym J. 2021; 151

30. Johns S, Poulsen T, Kane JJ, Windes WE, Ubic R, Karthik C. Formation of carbon nanostructures in nuclear graphite under high-temperature in situ electron-irradiation. Carbon. 2019; 143: 908-14.

31. Bai S, Yang N, Wang X, Gong F, Dong Z, Gong Y, et al. Ultrasmall Iron-Doped Titanium Oxide Nanodots for Enhanced Sonodynamic and Chemodynamic Cancer Therapy. ACS Nano. 2020; 14: 15119-30.

32. Wang S, Chen L, Wang J, Du J, Li O, Gao Y, et al. Enhanced-fluorescent imaging and targeted therapy of liver cancer using highly luminescent carbon dots-conjugated foliate. Mater Sci Eng: C. 2020; 116: 111233.

33. Jin $\mathrm{E}$, Yang $\mathrm{O}$, Ju CW, Chen $\mathrm{O}$, Landfester $\mathrm{K}$, Bonn $\mathrm{M}$, et al. A Highly Luminescent Nitrogen-Doped Nanographene as an Acid- and Metal-Sensitive Fluorophore for Optical Imaging. J Am Chem Soc. 2021; 143: 10403-12.

34. Lai IP-J, Harroun SG, Chen S-Y, Unnikrishnan B, Li Y-J, Huang C-C. Solid-state synthesis of self-functional carbon quantum dots for detection of bacteria and tumor cells. Sens Actuators B Chem. 2016; 228: 465-70.

35. Iravani S, Varma RS. Green synthesis, biomedical and biotechnological applications of carbon and graphene quantum dots. A review. Environ Chem Lett. 2020; 18: 703-27.

36. Li M, Chen T, Gooding JJ, Liu J. Review of Carbon and Graphene Quantum Dots for Sensing. ACS Sens. 2019; 4: 1732-48

37. Rhazouani A, Gamrani H, El Achaby M, Aziz K, Gebrati L, Uddin MS, et al. Synthesis and Toxicity of Graphene Oxide Nanoparticles: A Literature Review of In vitro and In vivo Studies. Biomed Res Int. 2021; 2021: 5518999.

38. Wu J, Li Z, Tan H, Du S, Liu T, Yuan Y, et al. Highly Selective Separation of Rare Earth Elements by Zn-BTC Metal-Organic Framework/Nanoporous Graphene via In situ Green Synthesis. Anal Chem. 2021; 93: 1732-9.

39. Tan $\mathrm{H}$, Zhang $\mathrm{X}, \mathrm{Li} \mathrm{Z}$, Liang $\mathrm{Q}, \mathrm{Wu} \mathrm{J}$, Yuan $\mathrm{Y}$, et al. Nitrogen-doped nanoporous graphene induced by a multiple confinement strategy for membrane separation of rare earth. iScience. 2021; 24: 101920. 
40. Tan H, Liu T, Zhang X, Shan Q, Chen J, Li Z, et al. Preparation of Vortex Porous Graphene Chiral Membrane for Enantioselective Separation. Anal Chem. 2020; 92: 13630-3.

41. Li Z, Tian L, Liu J, Qi W, Wu Q, Wang H, et al. Graphene Oxide/Ag Nanoparticles Cooperated with Simvastatin as a High Sensitive X-Ray Computed Tomography Imaging Agent for Diagnosis of Renal Dysfunctions. Adv Healthc Mater. 2017; 6: 1700413.

42. Wang X, Wu X, Lu Z, Tao X. Comparative Study of Time-Resolved Fluorescent Nanobeads, Quantum Dot Nanobeads and Quantum Dots as Labels in Fluorescence Immunochromatography for Detection of Aflatoxin B1 in Grains. Biomolecules. 2020; 10: 575

43. Pieper H, Chercheja S, Eigler S, Halbig CE, Filipovic MR, Mokhir A. Toxizität von Graphenoxid: Endoperoxide als Ursache. Angew Chem. 2016; 128: 413-6.

44. Longlong T, Xin Z, Wei Q, Dan L, Qiang J, Jin L, et al. The adsorption of water-soluble ionic liquids on graphene oxide of different oxygen content. RSC Adv. 2014; 4: 58536-45.

45. Chong $\mathrm{Y}, \mathrm{Ma} \mathrm{Y}$, Shen $\mathrm{H}, \mathrm{Tu} \mathrm{X}$, Zhou $\mathrm{X}, \mathrm{Xu} \mathrm{J}$, et al. The in vitro and in vivo toxicity of graphene quantum dots. Biomaterials. 2014; 35: 5041-8.

46. Hunter WM, Greenwood FC. Preparation of iodine-131 labelled human growth hormone of high specific activity. Nature. 1962; 194: 495

47. Yang K, Wan J, Zhang S, Zhang Y, Lee S-T, Liu Z. In vivo pharmacokinetics, long-term biodistribution, and toxicology of PEGylated graphene in mice. ACS nano. 2010; 5: 516-22.

48. Yan H, Xue Z, Xie J, Dong Y, Ma Z, Sun X, et al. Toxicity of Carbon Nanotubes as Anti-Tumor Drug Carriers. Int J Nanomed. 2019; 14: 10179-94.

49. Rostami-Tapeh-Esmaeil E, Golshan M, Salami-Kalajahi M, RoghaniMamaqani H. Synthesis of copper and copper oxide nanoparticles with different morphologies using aniline as reducing agent. Solid State Commun. 2021; 334-335. 